\title{
Evaluation of damping properties of composite mount using a five-element model with variable parameters
}

\author{
H. Andou ${ }^{1}$, T. Ohkado ${ }^{2}$, N. Tsujiuchi ${ }^{2} \&$ T. Koizumi ${ }^{2}$ \\ ${ }^{I}$ Test \& Validation Department, Shin Caterpillar Mitsubishi Ltd, Japan \\ ${ }^{2}$ Department of Mechanical Engineering, Doshisha University, Japan
}

\begin{abstract}
A mount is designed that combines air-spring with viscous fluid. In this mount, air-spring generates the dynamic stiffness, and the shearing of viscous fluid generates the loss stiffness. The conceptual purposes are to adjust a neutral position easily by changing the pressure of the air-spring and to prevent a rapid drop of the damping performance from the stationary characteristics in the case of excess load.

The purpose of this research is to evaluate damping properties of the composite mount. First, the dynamic characteristics of the composite mount are obtained by excitation tests of the mount itself. But the characteristics might not be identified by the standard mechanical model accuracy because they depend on not only excitation frequency but also amplitude. So the nonlinear dynamic characteristics are identified using the five-element model with a variable parameter. Finally, the composite mount is compared with the viscous mount by simulation using the identified model, and the damping performance is quantitatively indicated.
\end{abstract}

Keywords: mount, damping properties, five-element model, Maxwell model.

\section{Introduction}

The ride comfort for the hydraulic excavator has been strongly demanded every year. Not only the structural durability of the cab but also the improvement of cab vibration is an important requirement. The viscous mount is widely used as a mount to reduce the cab vibration, which is shown in fig. 1 . The mount mainly 
consists of the annular rubber insulator, the damping plate, and sealed high viscosity oil. Viscous mount combines the damping force from the pressure difference of the viscous fluid with the elastic force by the rubber insulator. The viscous mount demonstrates a damping performance greater than a conventional rubber mount. Therefore, the viscous mount helps to extend the durability of the cab structure and to reduce the cab vibration.

However, in order to reduce the cost, the same mount is installed on various sized vehicles. Moreover, the designed upper and lower stroke in the vertical direction is not equal i.e. the neutral position is not in the centre of the stroke because of the difference in the weight. When the weight is heavy, the neutral position is depressed down. Then, the displacement might be beyond the designed stroke range, the rubber insulator having a small damping capacity absorbs the load as stopper. Thus, an operator experiences a strong shock load due to the stopper rubber insulator. Besides, the high viscosity oil can hardly flow through a narrow clearance at a large load, and the cab receives the compressive repulsion power of oil because of sealed viscous oil thereby restricting the damping ability. In addition, it is difficult to obtain an arbitrary damping performance separately, because damping stiffness increases with increasing dynamic stiffness [1]. To overcome these problems, it is required that the viscous mount responds according to various applications of hydraulic excavators.

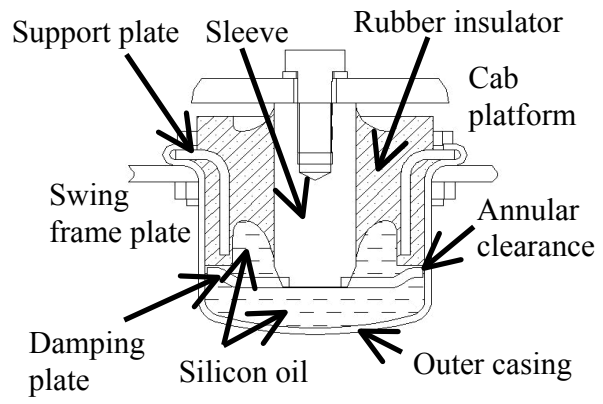

Figure 1: Viscous mount.

\section{Structure of the composite mount}

A mount was invented that combines the shear force due to viscous fluid with elastic force due to air-spring. Fig.2 shows the structure and design dimensions of the composite mount that is used for this research. The outer tube, in which high viscosity oil is filled, is equipped in the air-spring. In order to perform the shear viscous damping, the annular clearance for the high viscosity oil is prepared by inserting the inner tube with a 45 degree chamfer at the inside edge. The initial neutral position for the designed mount can be independently adjusted by pressure of the air-spring depending on the weight [2]. The loading capacity 
per one mount is $8.1 \mathrm{kN}$. In the case that a cab is supported by four mounts, a cab weight of up to $32.4 \mathrm{kN}$ is allowed. In addition, viscous oil is not sealed and can flow at a large load. Therefore, in the case of a large load, it may not experience the repulsion force of oil, and the damping ability might perform well enough.

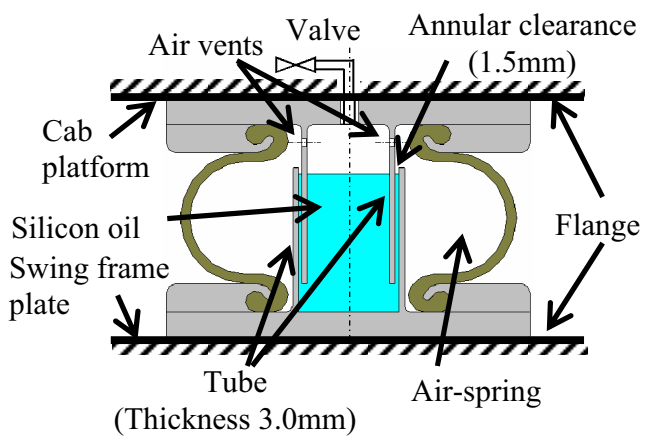

Figure 2: $\quad$ Composite mount.

An air valve is installed on the upper flange to easily change the pressure of the air-spring by an external device. The air vents are located on the upper tube. The silicon oil, polymeric fluid, is used as high viscosity oil.

When the excitation at the vertical direction is applied between the upper and lower flange for the composite mount, the air-spring deflects and the inner tube moves in viscous oil. The air-spring generates the elastic force, while the annular clearance generates the viscous shear force as the damping force on wall. The elasticity force can be adjusted by changing the pressure of the air-spring. The damping force can be designed by changing the viscosity or volume of the oil respectively.

\section{Dynamic characteristics}

\subsection{Excitation test}

Fig. 3 shows the test device for evaluating the dynamic characteristics of the composite mount in the vertical direction. The upper surface of the composite mount was fixed on the jig, and the shaker excited the lower flange.

At first, the specified pressure of the air-spring was adjusted through the air valve. The swept sinusoidal excitation test under constant displacement was performed by the feedback control to the displacement. The load cell measured the excitation force, and the laser displacement sensor measured the displacement.

All test cases as shown below were performed.

Frequency (sweep rate is $0.5 \mathrm{~Hz} / \mathrm{s}$ ):

4 to $12 \mathrm{~Hz}$ on sweep

Displacement:

$1.0,2.0,3.0 \mathrm{~mm}$

Pressure of air-spring (gage pressure):

$1.0,1.5,2.0 \mathrm{~atm}$

Viscosity of viscous oil: 


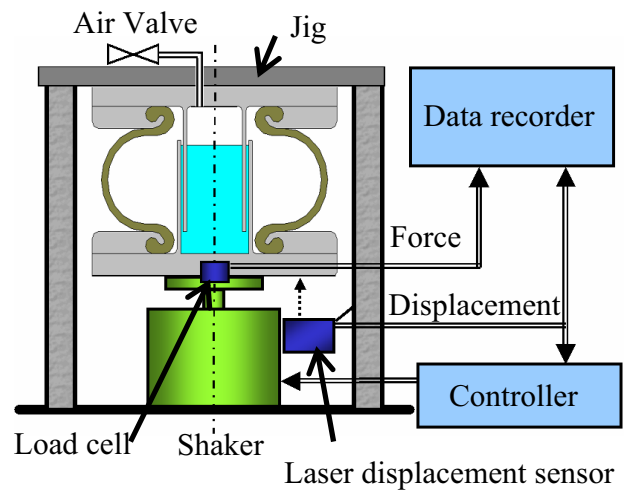

Figure 3: Excitation test.

The frequency range from $4-8 \mathrm{~Hz}$ is more sensitive to a human body, according to ISO2631 [3]. So, the excitation test beyond $12 \mathrm{~Hz}$ is omitted in this paper.

\subsection{Calculation of the dynamic characteristics}

Using FFT, eqn. (1) and eqn. (2) respectively show the dynamic stiffness and the loss stiffness [4].

$$
\begin{gathered}
k_{d}=\frac{\operatorname{Re}(\widetilde{F}) \operatorname{Re}(\widetilde{x})+\operatorname{Im}(\widetilde{F}) \operatorname{Im}(\widetilde{x})}{\operatorname{Re}(\widetilde{x})^{2}+\operatorname{Im}(\tilde{x})^{2}} \\
c \omega=\frac{\operatorname{Im}(\widetilde{F}) \operatorname{Re}(\widetilde{x})-\operatorname{Re}(\widetilde{F}) \operatorname{Im}(\widetilde{x})}{\operatorname{Re}(\widetilde{x})^{2}+\operatorname{Im}(\widetilde{x})^{2}}
\end{gathered}
$$

where Re is the real part, Im is the imaginary part, $\widetilde{F} \mathrm{~N}$ is the complex force and $\widetilde{x} \mathrm{~m}$ is the complex displacement.

For dynamic stiffness, the dependency on frequency, displacement and viscosity of viscous oil doesn't appear. Fig. 4 shows the dynamic stiffness against pressure. The dynamic stiffness increases with an increase in the pressure of the air-spring. Thus the dynamic stiffness depends only on pressure of the air-spring.

Fig. 5 shows the loss stiffness against frequency for the oil viscosity 9.75 $\mathrm{Ns} / \mathrm{m}^{2}$ and $97.50 \mathrm{Ns} / \mathrm{m}^{2}$.

Fig.5(a) shows that the loss stiffness for the oil viscosity $9.75 \mathrm{Ns} / \mathrm{m}^{2}$ increases with frequency and doesn't depend on displacement. Fig.5(b) indicates that the loss stiffness for the oil viscosity $97.50 \mathrm{Ns} / \mathrm{m}^{2}$ increases with frequency, more than that of the oil viscosity $9.75 \mathrm{Ns} / \mathrm{m}^{2}$. Also, it slightly increases on the upper frequency range and decreases with increasing displacement.

As the results show, the damping property of the composite mount can be independently designed by changing the oil viscosity regardless of the dynamic stiffness. This is a significant result [5]. 


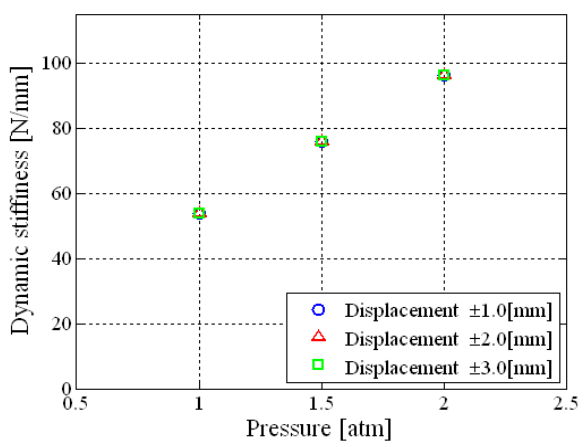

Figure 4: $\quad$ Dynamic stiffness vs. pressure.

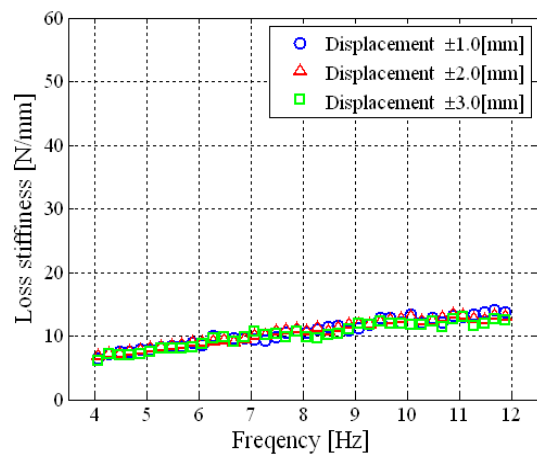

a) Viscosity: $9.75 \mathrm{Ns} / \mathrm{m}^{2}$

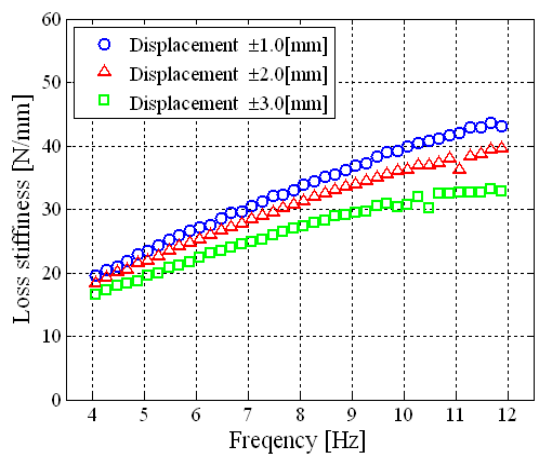

b) Viscosity: $97.50 \mathrm{Ns} / \mathrm{m}^{2}$

Figure 5: Loss stiffness vs. frequency in the composite mount.

\section{Simulation model of the composite mount}

In order to simulate the response behaviour of the composite mount, modelling of the dynamic characteristics is needed. However a constant spring-damper element can hardly express the above-mentioned nonlinear characteristics with a high accuracy [6]. Then, the dynamic stiffness and the loss stiffness with the dependencies on the frequency and the displacement are identified by the five-element Maxwell model with variable parameters [7].

Fig.6 shows the five-element Maxwell model with variable parameters. The dynamic stiffness $k_{d}(f)$ and the loss stiffness $c \omega(f)$ of the five-element Maxwell model with the variable parameter as depending on the velocity shown in fig. 6 are defined in eqns. (3) and (4).

$$
k_{d}(f)=k_{0}+\frac{4 \pi^{2} f^{2} k_{1} c_{1}{ }^{2}}{k_{1}{ }^{2}+4 \pi^{2} f^{2} c_{1}{ }^{2}}+\frac{4 \pi^{2} f^{2} k_{2} c_{2}{ }^{2}}{k_{2}{ }^{2}+4 \pi^{2} f^{2} c_{2}{ }^{2}}
$$




$$
c \omega(f)=\frac{2 \pi f k_{1}{ }^{2} c_{1}}{k_{1}{ }^{2}+4 \pi^{2} f^{2} c_{1}{ }^{2}}+\frac{2 \pi f k_{2}{ }^{2} c_{2}}{k_{2}{ }^{2}+4 \pi^{2} f^{2} c_{2}{ }^{2}}
$$

where spring rates are $k_{0}, k_{1}$ and $k_{2} \mathrm{~N} / \mathrm{m}$, damping coefficients are $c_{1}$ and $c_{2}$ $\mathrm{Ns} / \mathrm{m}$ and a frequency is $f \mathrm{~Hz}$.

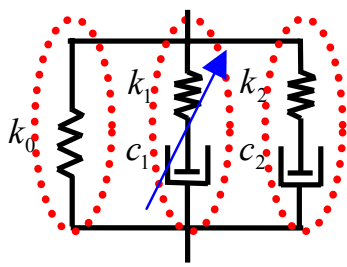

\section{Unit1 Unit2 Unit3}

Figure 6: $\quad$ Five-element Maxwell model depending on velocity.

The relationships between the spring rate and the damping coefficient for each unit are defined in eqns. (5) and (6) to easily identify the parameters.

$$
\begin{aligned}
& c_{1}=\frac{k_{1}}{2 \pi \alpha_{1}} \\
& c_{2}=\frac{k_{2}}{2 \pi \alpha_{2}}
\end{aligned}
$$

Then, the coefficients $\alpha_{1}$ and $\alpha_{2}$ mean the extreme value of the loss stiffness.

The maximum velocity $v \mathrm{~m} / \mathrm{s}$ is expressed by the excitation displacement in eqn. (7).

$$
v=2 \pi f D
$$

where the amplitude of displacement is $D \mathrm{~m}$.

The five-element Maxwell model parameters including variables are identified for each displacement at the air pressure $1.0 \mathrm{~atm}$, the viscosity 97.50 $\mathrm{Ns} / \mathrm{m}^{2}$ by the Gauss-Newton method, a nonlinear least-squares approximation, using eqns. (3) and (4). The variable parameter $k_{1}$ is defined as the function of the maximum velocity in the eqn. (8) based on the measured dynamic characteristics.

$$
k_{1}=p_{1} v^{2}+p_{2} v+p_{3}
$$

Table 1 shows the results of the identified parameters. The identified results of the dynamic characteristics are shown in fig. 7.

Table 1: $\quad$ Five-element Maxwell model with variable parameter.

\begin{tabular}{|c|c|c|c|c|c|c|}
\hline $\mathrm{k}_{0}$ & $\mathrm{p}_{1}$ & $\mathrm{p}_{2}$ & $\mathrm{p}_{3}$ & $\mathrm{k}_{2}$ & $\alpha_{1}$ & $\alpha_{2}$ \\
\hline 53512 & 1461000 & -1229000 & 288800 & 1001590 & 100 & 500 \\
\hline
\end{tabular}




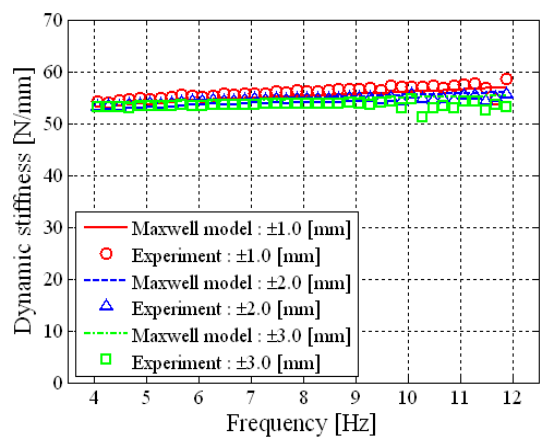

(a) Dynamic stiffness

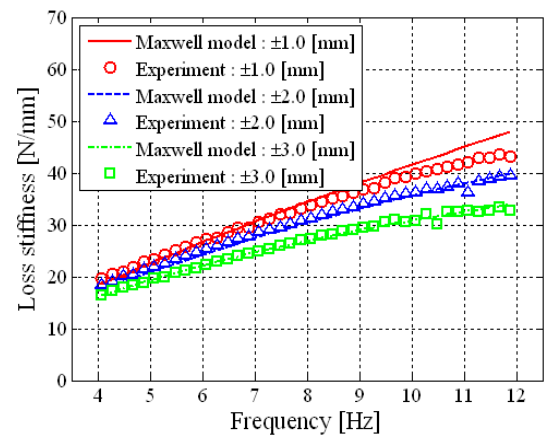

(b) Loss stiffness

Figure 7: Comparison of the Maxwell model with the experiment.

Table 2: $\quad$ Error of modelling.

\begin{tabular}{|c|c|c|c|}
\hline \multirow{2}{*}{} & \multicolumn{3}{|c|}{ Displacement $\mathrm{mm}$} \\
\cline { 2 - 4 } & \pm 1.0 & \pm 2.0 & \pm 3.0 \\
\hline Dynamic stiffness & $1.67 \%$ & $1.13 \%$ & $2.35 \%$ \\
\hline Loss stiffness & $5.15 \%$ & $2.23 \%$ & $1.35 \%$ \\
\hline
\end{tabular}

Moreover, the error is calculated using eqn. (9).

$$
S=\sqrt{\frac{\sum_{i=1}^{m}\left(E_{i}-F_{i}\right)^{2}}{\sum_{i=1}^{m}\left(E_{i}\right)^{2}}}
$$

where the number of data is $m$, the measured dynamic characteristic for each frequency at the specified displacement is $E_{i}$ and the identified dynamic characteristic is $F_{i}$. The errors are shown in Table 2.

The dynamic stiffness and the loss stiffness of the composite mount could be identified by the accuracy of $5.15 \%$ error or less, using the five-element Maxwell model with the variable parameter due to the vibration velocity.

\section{Performance evaluation of composite mount}

In this section, the vibration absorption performance is quantitatively evaluated by the identified Maxwell model using the actual excitation at a digging condition for a hydraulics excavator. In addition, the profit of the composite mount is referred comparing with the performance of the viscous mount. 


\subsection{Simulation model for performance evaluation}

The cab is supported by four viscous mounts on the swing frame. The various external excitations for a digging and travelling machine are transferred to the cab through the swing frame and the mount. The schematic diagram is shown in fig.8. The swing frame and the cab itself are the complex metal structures with the flexibility. The response vibration is calculated by the simple model, in which the feature of the composite mount can be easily understood.

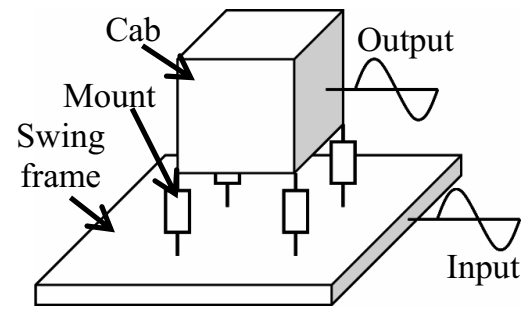

Figure 8: Schematic diagram.

\subsection{Response result}

Applying the external acceleration for 16 seconds where the sampling frequency is $512 \mathrm{~Hz}$, the response acceleration and the displacement are calculated by the cab simulation model including the identified Maxwell model. The properties of the identified Maxwell model are updated by each calculated mount velocity during the transient response analysis. In order to clarify the characteristics of the composite mount, the responses are compared with one of the viscous mounts.

The amplification between the input acceleration and the response acceleration is calculated from the power spectrum obtained by applying FFT to the acceleration data of the input and output. The result is shown in fig.9. The amplification of the composite mount indicates the very high magnification, 13.4 at $2.8 \mathrm{~Hz}$. The amplification of the viscous mount indicates 3.7 at $4.8 \mathrm{~Hz}$. The profit of the composite mount is in the frequency range over $3.7 \mathrm{~Hz}$.

The response displacement between the upper and lower flanges of the mount is relatively calculated. The result is shown in fig.10. The dashed line at $\pm 3.0 \mathrm{~mm}$ displacement indicates the limit of the viscous mount in the viscous damping stroke as a reference.

The maximum displacement of the composite mount was $\pm 27 \mathrm{~mm}$ greater than $\pm 10 \mathrm{~mm}$ of the viscous mount. Basically, the mount with the viscous oil demonstrates the damping performance by converting the acceleration into the displacement. The response acceleration and displacement are usually in contrast. For the development of the cab mount, the displacement is the constraint condition. Therefore, if the composite mount is used as a cab mount, it's necessary to examine the layout, which allows the displacement over \pm 27 mm. 


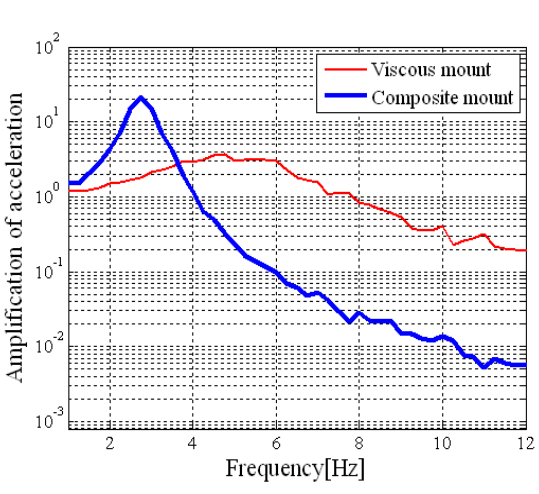

Figure 9: Amplification of acceleration.
Limit of relativity displacement:

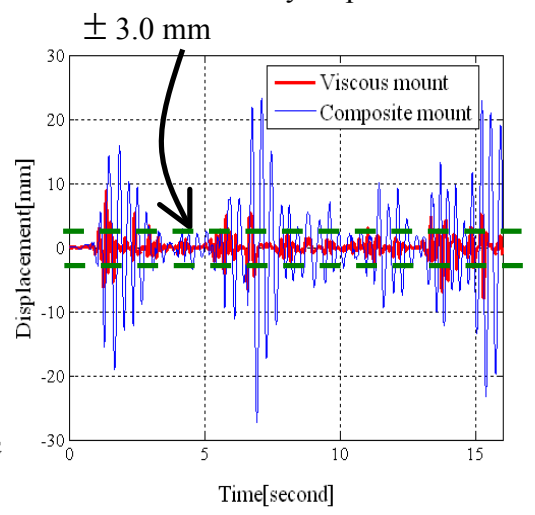

Figure 10: Relative displacement.

Using the five-element Maxwell model with the variable parameter, which identifies the dynamic characteristics of the mount with nonlinearity with a high accuracy, the response simulation can be quantitatively performed. That is, the mount specifications can be iteratively tuned using the process mentioned in this paper.

\section{Conclusion}

The following conclusions can be demonstrated.

- The composite mount combining the viscous shear force due to viscous fluid with the elastic force due to air-spring was designed as the mount to overcome the concerns of the currently used viscous mount.

- For the dynamic characteristics of the composite mount, the dependence on frequency, displacement, pressure of the air-spring and viscosity of viscous oil were clarified by the excitation test.

- The dynamic stiffness and the loss stiffness of the composite mount could be identified by the accuracy of $5.15 \%$ error or less, using the five-element Maxwell model with the variable parameter due to the vibration velocity.

- Using the five-element Maxwell model with the variable parameter, which identifies the dynamic characteristics of the mount with nonlinearity with a high accuracy, the response simulation can be quantitatively performed.

\section{References}

[1] Hiroaki Andou, Takayuki Koizumi, Nobutaka Tsujiuchi and Toshiyuki Shibayama, Numerical Model of Viscous Mount for Hydraulic Excavator, IMAC-XXIII, 2004 
[2] Neil Sclater, Nicholas P. Chironis, Mechanisms and Mechanical Devices Sourcebook third edition, The McGraw-Hill Companies, pp186-187, 2001

[3] Dong-Wook Lee, Choo-Ho Kim, Soon-Woo Han, and Sung-Chul Lee, Improvement of Ride Comfort of the Excavator, SAE Technical paper series

[4] Hiroaki Andou, Takayuki Koizumi, Nobutaka Tsujiutchi and Toshiyuki Shibayama, Using FFT to compute frequency-dependent dynamic characteristics, APVC, 2005

[5] Hiroaki Andou, Nobutaka Tsujiuchi, Takayuki Koizumi and Takudou Ohkado, Dynamic characteristics of a mount combining viscous fluid with air-spring, SAE, 2007

[6] S.W.Park, Analytical modeling of viscoelastic dampers for structural and vibration control, International Journal of Solids and Structures, vol.38, pp.8065-8092, 2001

[7] Yoshihiro Hamazaki, Satoshi Yonezawa and Yoshio Inoue, Study of Dynamic Behavior of Highly Viscous Fluid Mount (Modeling of Frequency and Displacement Dependency), JSME, vol.62, 1996 